\title{
Estado poblacional del ganado Reyna en Nicaragua: distribución, manejo, producción y cualidades
}

\section{Reyna livestock population status in Nicaragua: distribution, management, production and qualities}

\author{
Marlon Hernández-Baca \\ Facultad de Ciencia Animal, Universidad Nacional Agraria.
}

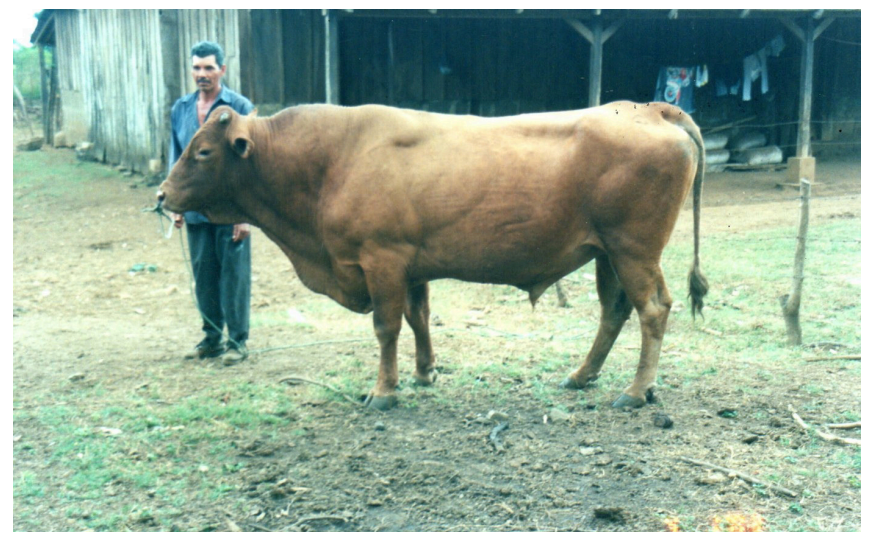

\section{RESUMEN}

El presente estudio se realizó en colaboración con el organismo italiano CROCEVIA, y en él se pretendió determinar el nivel poblacional, distribución geográfica, elementos de manejo, utilización y características del ganado criollo Reyna. Se determinó que esta raza está presente en once de los dieciséis departamentos del país, alcanzando una población total de 1963 cabezas, de las cuales 1184 corresponden al hato puro y 779 al hato cruzado, siendo los departamentos de Rivas y Boaco los que alcanzaron la mayor población de hato puro. Los sistemas donde se explota esta raza, principalmente son de doble propósito, con alimentación basada en el pastoreo, que en algunos casos se acompaña de suplementación durante el ordeño. Se identificaron 20 especies de pastos en las fincas donde se explota esta raza. La producción de leche en el hato puro se obtiene por ordeño doble o sencillo alcanzando en el primer caso niveles de cuatro a cinco $\mathrm{kg}$ en invierno y de cinco a seis $\mathrm{kg}$ en verano, y cuando se realiza un solo ordeño, $4.5 \mathrm{~kg}$ y $5.5 \mathrm{~kg}$ en invierno y verano respectivamente. En el hato cruzado prevalece un solo ordeño con producciones que oscilan entre cuatro y $11 \mathrm{~kg}$ en invierno y de tres a 13 $\mathrm{kg}$ en verano. De los cruces raciales en los que participa el Reyna se registraron 23, con el predominio de media sangre y un cuarto Reyna en combinación con seis razas. En relación a sus cualidades se registraron 18 bondades y siete defectos señalados por los productores Palabras clave: Población, distribución, manejo, calidad, cualidades, defectos.

\section{ABSTRACT}

This study was conducted in collaboration with the Italian agency CROCEVIA, and its purpose was to determine the population level, geographical distribution, management elements, use and characteristics of Reyna Creole cattle. This breed is present in eleven of the sixteen departments in our country, reaching a total population of 1963 heads, of which 1184 corresponded to pure herd and 779 to cross herd, the departments of Rivas and Boaco have the largest population of pure herd. Systems where this breed is exploited, are mostly double purpose, based on grazing, which in some cases is accompanied by food supplementation during milking, achieving identified 20 different grass species on farms where this breed is exploited. Milk production in the pure herd is obtained by double or single milking, reaching in the first case $4 \mathrm{~kg}$ to $5 \mathrm{~kg}$ in winter and in summer $5 \mathrm{~kg}$ to $6 \mathrm{~kg}$ and in one milking $4.5 \mathrm{~kg}$ to $5.5 \mathrm{~kg}$ in winter and summer respectively. In the herd crossed prevailing a single milking, with production ranging from $4 \mathrm{~kg}$ to $11 \mathrm{~kg}$ in winter and in summer $3 \mathrm{~kg}$ to $13 \mathrm{~kg}$. Racial crosses in which participates Reyna 23 were reported, with the predominance of the half blood and a fourth Reyna, in combination with 6 different races. This breed has 18 attributes and 7 defects identified by producers, having more benefits than flaws. Key words: Population, distribution, management, production, qualities, defects. 
$\mathrm{E}$ 1 bovino criollo americano desciende directamente de los animales que llegaron en el segundo viaje de Colón en 1493, a la isla de Santo Domingo o La Española. Según Rouse (1977), citado por Primo (1992), derivaron del Bos primigenius.

En Centroamérica, el ganado llegó a Castillo de Oro, hoy Panamá, de donde fueron traídos a Nicaragua por Francisco Hernández de Córdoba entre los años 1524 y 1526 (Salvatierra (1939) citado por Guerrero y Soriano, 1992), (Esgueva, 2006) expidiéndose su partida de nacimiento en la ciudad de Burgos España el 20 de diciembre de 1527, cuando le autorizan a Pedrarias Dávila trasladar toda su hacienda desde Panamá hacia Nicaragua, fundando tres zonas ganaderas, la de Chinandega, una segunda en Granada y la tercera en el viejo Chontales.

No se tienen datos exactos de la cantidad de ganado que existía en el país durante la colonia, sin embargo, Incer (1969) señala que las exportaciones de Nicaragua en 1730 llegaron a 14000 cabezas, en 1731 a 18000 , en 1764 a 52000 y en 1797 alcanzaron las 10159 reses. (Guerrero y Soriano, 1992) hacen referencia de Fray Agustín Morel de Sta. Cruz (1751 y 1752), señalando que la población de ganado vacuno en la zona de Chontales era de aproximadamente 40000 cabezas y en la zona de Granada alcanzaba las 25000 reses; sin referirse a la población de ganado correspondiente a la zona de Chinandega.

El primer dato de la cantidad de ganado existente en el país es señalado por Levy (1976), citado por Incer, (1969) quien señala que en 1871 la población ganadera del país alcanzaba el millón de reses.

Entre 1890 y 1900 se inicia la sustitución del ganado criollo, con la importación de toros Holstein; para 1920 en Rivas ya se cruzaba Brahman con criollo (FAO 2005). En 1940 se introduce la raza Pardo Suizo y con la modernización de la infraestructura de matanza entre 1950 y 1960 inicia la debacle del ganado criollo que constituía la mayor proporción de la masa ganadera total del país, pues se inició de manera intensa el apareamiento con cebúes, resultando en un tipo de animal con buen vigor hibrido que entusiasmo a los ganaderos a realizar repetidamente el cruce, y terminar absorbiendo a los criollos en su gran mayoría, obteniéndose gran cantidad de animales de la raza Sta. Gertrudis, con el supuesto propósito de mejorar la calidad y el rendimiento de nuestra ganadería (Incer, 1969).

Después de 143 años de la última cifra citada por Levi (1976) sobre la población del criollo, el presente estudio pretende ser un punto de partida en cuanto a la actualización y conocimiento de la distribución geográfica, población y otros elementos relativos al manejo, utilización y características del ganado criollo Reyna en Nicaragua, representante único de los tipos de ganado bovino traídos por los españoles.

\section{MATERIALES Y MÉTODOS}

Se utilizaron metodologías cualitativas y cuantitativas para obtener la información base del estudio. Los métodos de informantes claves y rastreo por municipios, permitieron conocer la ubicación de las zonas y productores con explotación del criollo Reyna, de manera que comenzando con los propietarios de los hatos puros de Rivas, se inició el seguimiento uno a uno a las personas que realizaron compras en estos hatos y así sucesivamente cada productor fue dando la ubicación y pistas de otros criadores. En el ministerio agropecuario y forestal se obtuvo muy poca información.

Una encuesta base se elaboró para el abordaje de los productores, en la cual se incluyeron puntos relacionados a la ubicación general, extensión y nombre de la finca, datos generales del propietario, número de animales Reyna puro o en cruces, grupos raciales con participación del crio1lo, tipo de ordeño, producción de leche, tipo de alimentación y pastizales, bondades y defectos del ganado criollo.

Un muestreo aleatorio de la calidad de la leche se realizó en los hatos puros en el periodo de invierno correspondiente con el tiempo en que se realizó el estudio, obteniéndose muestras por estratos de vacas con diferentes números de partos y cuyos análisis se realizaron a través de métodos aprobados por la AOAC (association of analytical communities).

La cuantificación de la información se realizó a partir de la consolidación de los datos numéricos registrados en la encuesta, la que se procesó utilizando $\mathrm{Mi}$ crosoft Excel y Word, obteniéndose los numerales de población por cada departamento y población total del hato, y de la misma manera se consolidó la información cualitativa teniendo como base los detalles de la encuesta.

En las determinaciones de producción por periodo del año y calidad de la leche se aplicó estadística descriptica y de esta manera se obtuvieron los promedios representativos.

\section{RESULTADOS Y DISCUSIÓN}

Distribución del ganado criollo puro y cruzado. Once son las principales zonas donde se desarrolla la crianza de hatos puros y cruzados del criollo Reyna, estas son: Rivas, Granada, Masaya, Carazo, Managua, León, Chinandega, Boaco, Chontales, RAAS, y Matagalpa.

En el departamento de Rivas, los hatos se asientan en los municipios de Tola, Potosí, San Jorge, San Juan del Sur y Belén, constituyendo el departamento con mayor distribución en cuanto al número de municipios en los cuales se distribuyen los hatos.

En Granada, mayoritariamente encontramos hatos cruzados en los municipios de Nandaime y Diriomo. En Masaya hay presencia de hatos puros y cruzados en los municipios de Masatepe y los Altos. Carazo presenta también algunos hatos cruzados en el municipio de Jinotepe. 
En Managua sobresale el hato puro de la Universidad Nacional Agraria. En León se localizó un hato cruzado en el municipio de Telica y en Chinandega se ubicaron hatos puros y cruzados en los municipios del Viejo y el Realejo.

En el departamento de Boaco se encontraron hatos puros en el municipio del mismo nombre y en $\mathrm{Ca}$ moapa. Chontales presentó hatos cruzados en los municipios de Juigalpa y Acoyapa. En la RAAS, se localizó un hato cruzado en el municipio de Muelle de los Bueyes, finalizando en el departamento de Matagalpa, se ubicaron hatos cruzados en el municipio de Muy muy.

Si tenemos en cuenta que el Reyna puro y sus cruces están presente en once de los dieciséis departamentos del país, a pesar de presentar poblaciones muy reducidas, prácticamente se encuentra en la mayor parte del territorio nacional, aun cuando la mayoría de los ganaderos consideran una extravagancia el tener esta raza de ganado; lo que puede atribuirse al desconocimiento de las virtudes del Reyna en cruces con otras razas de alta especialización, y a la falta de una política adecuada de difusión de la raza por parte de la asociación de criadores del hato puro.

Manejo y alimentación del hato. En las zonas señaladas, los hatos son manejados en sistemas de producción muy simples cuya alimentación se basa fundamentalmente en el pastoreo y en algunos casos muy particulares, se les suministra a las vacas en ordeño un suplemento consistente en concentrados elaborados en las fincas o simplemente gallinaza más pequeñas porciones de concentrado. Estas prácticas son comunes sobre todo en los hatos ubicados en las zonas de Rivas y Masaya (Masatepe), ya que en las áreas restantes la alimentación recae únicamente sobre los pastizales en los que los animales pacen ad libitum, junto al consumo de sales minerales.
En el cuadro 1 se presentan 20 especies de pastos encontradas en las fincas donde se explota el Reyna.

Ordeño, producción y calidad de leche del Reyna. En las haciendas de hato puro se realizan tres formas de ordeño, que van desde el ordeño doble (cinco am y tres pm), el ordeño único por la mañana (4:30 am) y el ordeño vespertino $(2: 30 \mathrm{pm})$. Los dos primeros se caracterizan por utilizar el apoyo del ternero, no así en el último, en el que las vacas son ordeñadas de manera libre. Una gran excepción se encontró en el hato ubicado en Masatepe, municipio de Masaya, en el cual se practica doble ordeño, los machos se sacrifican a los ocho días y se separan las hembras de las madres a los cuatro meses, a partir del cual se les suministra pasto picado más concentrado.

En los restantes hatos predominan los sistemas de ordeños tradicionales, caracterizados por una sola extracción, el apoyo del ternero y la utilización de un amamantamiento restringido.

En cuanto a la producción de leche del hato puro, en doble ordeño esta oscila entre cuatro y cinco $\mathrm{kg}$ en invierno y hasta cinco a $6.5 \mathrm{~kg}$ en verano, en ordeño único estas producciones alcanzan entre 4.5 y $5.5 \mathrm{~kg}$ en invierno y verano respectivamente. Este comportamiento productivo tiene diferentes tendencias en el hato cruzado, debido a la diversidad y niveles de razas que pueden intervenir en dichos cruces, pero de manera general oscilan entre cuatro y $11 \mathrm{~kg}$ en invierno y de tres a $13 \mathrm{~kg}$ en verano.

En cuanto a la calidad de la leche del Reyna puro en las muestras aleatorias colectadas en el periodo lluvioso, se registran niveles de proteína muy variados según el número de partos y el tipo de alimentación, pero de manera general los contenidos nutricionales se presentan en el cuadro 2 .

Cuadro 1. Especies de pastos utilizadas en las fincas donde se explota el Reyna

\begin{tabular}{llll}
\hline \multicolumn{1}{c}{ Nombre común } & \multicolumn{1}{c}{ Nombre científico } & Nombre común & \multicolumn{1}{c}{ Nombre científico } \\
\hline Alemán & Echinochloa polystacha Hitchc & Gamalote & Axonopus scoparius Hitche \\
Angleton & Dichantium aristatum Hubbard & Grama & Paspalum conjugatum Berg \\
Brachiaria brizantha & Brachiaria brizantha Stapf & Guinea & Panicum máximum Jacq \\
Brachiaria decumbens & Brachiaria decumbens Stapf & India & Panicum máximum \\
Brachiaria dictoneura & Brachiaria dictyoneura Stapf & Jaragua & Hyparrhenia rufa Stapf \\
Colonial & Panicum máximum & Kutzu & Pueraria phaseoloides \\
Caña japonesa & Saccharum sinense Roxb & Para & Brachiaria mutica \\
Elefante & Pennisetum purpureum Schumach & Taiwan & Pennisetum sp \\
Estrella & Cynodon nlemfuensis & Tanzania & Panicum máximum \\
Gamba & Andropogon gayanus Kunth & Tifton & Cynodon sp \\
\hline
\end{tabular}


Cuadro 2. Calidad de la leche del hato puro Reyna

\begin{tabular}{lccc}
\hline N de parto & $\begin{array}{c}\text { Sólidos totales } \\
(\%)\end{array}$ & $\begin{array}{c}\text { Proteína } \\
(\%)\end{array}$ & $\begin{array}{c}\text { Grasa } \\
(\%)\end{array}$ \\
\hline Primero & 11.34 a 14.36 & 3.03 a 4.16 & 2.2 a 4.5 \\
Segundo & 10.48 a 13.24 & 2.86 a 3.75 & 2.1 a 4.0 \\
Tercero & 10.75 a 13.68 & 2.98 a 3.89 & 1.8 a 4.4 \\
Cuarto & 10.58 a 13.60 & 3.25 a 4.10 & 2.0 a 4.3 \\
Quinto & 10.78 a 13.98 & 3.14 a 3.89 & 2.2 a 4.6 \\
Sexto & 12.23 a 12.93 & 3.11 a 3.53 & 3.5 a 3.8 \\
Séptimo & 10.15 a 12.28 & 2.80 a 3.19 & 2.5 a 3.2 \\
Octavo & 12.62 a 13.15 & 3.14 a 3.71 & 3.6 a 4.0 \\
\hline
\end{tabular}

Cruces en los que ha participado el Reyna. En el cuadro 3 se pueden observar los múltiples cruces en los que se ha utilizado el ganado Reyna. Cada uno de los cruces indicados ha sido determinado por los criterios e intereses de cada productor, quienes han incorporado un determinado porcentaje de sangre Reyna a sus animales sobre la base de su experiencia y resultados obteni- dos. Sin embargo, el media sangre y 1/4 Reyna, son los que predominan en los cruces reportados por los ganaderos.

Población del ganado Reyna puro y cruzado. Como ya señalamos el Reyna tanto puro como en cruces está presente en once de los dieciséis departamentos de Nicaragua, sin embargo, sus poblaciones son muy irregulares de una zona a otra, encontrándose una población total de 1184 cabezas de animales puros y 779 en los diversos cruces. Los detalles por departamentos se pueden observar en el siguiente cuadro.

Cuadro 4. Población del ganado Reyna, hato puro y cruzado

\begin{tabular}{cccc}
\hline $\mathrm{N}^{\circ}$ & Departamento & Hato puro & Hato cruzado \\
\hline 1 & Rivas & 482 & 384 \\
2 & Granada & 4 & 90 \\
3 & Masaya & 156 & 20 \\
4 & Carazo & 2 & 51 \\
5 & Managua & 79 & 10 \\
6 & León & 0 & 100 \\
7 & Chinandega & 24 & 13 \\
8 & Matagalpa & 1 & 48 \\
9 & Boaco & 435 & 15 \\
10 & Chontales & 1 & 33 \\
11 & RAAS & 0 & 15 \\
\hline Total & & 1184 & 779 \\
\hline
\end{tabular}

Cuadro 3. Cruces con participación del ganado Reyna reportados por los productores

\begin{tabular}{clll}
\hline $\mathrm{N}^{\circ}$ & \multicolumn{1}{c}{ Cruce } & $\mathrm{N}^{\circ}$ & \multicolumn{1}{c}{ Cruce } \\
\hline 1 & $1 / 2$ Reyna $+1 / 4$ P.S $+1 / 4$ Brahaman & 13 & $1 / 4$ Reyna $+1 / 2$ Simmental $+1 / 8$ Brahman $+1 / 8$ PS \\
2 & $1 / 2$ Reyna $+1 / 6$ Holstein $+1 / 6$ PS $+1 / 6$ Brahman & 14 & $1 / 4$ Reyna $+1 / 2$ PS $+1 / 4$ Brahman \\
3 & $1 / 2$ Reyna $+1 / 4$ Holstein $+1 / 4$ Brahman & 15 & $1 / 4$ Reyna $+3 / 4$ Jersey \\
4 & $1 / 2$ Reyna $+1 / 4$ Jersey $+1 / 4$ PS & 16 & $1 / 4$ Reyna $+3 / 4$ Holstein \\
5 & $1 / 2$ Reyna $+1 / 2$ PS & 17 & $1 / 4$ Reyna $+3 / 8$ PS $+3 / 8$ Jersey \\
6 & $1 / 2$ Reyna $+1 / 2$ Holstein & 18 & $1 / 4$ Reyna $+1 / 4$ PS $+1 / 2$ Gyr \\
7 & $1 / 2$ Reyna $+1 / 2$ Brahman & 19 & $3 / 4$ Reyna $+1 / 4$ PS \\
8 & $1 / 2$ Reyna $+1 / 2$ Jersey & 20 & $3 / 4$ Reyna $+1 / 4$ Brahman \\
9 & $1 / 2$ Reyna $+1 / 4$ P.S $+1 / 4$ Gyr & 21 & $3 / 4$ Reyna $+1 / 4($ Jersey + PS + Brahman $)$ \\
10 & $1 / 4$ Reyna $+1 / 2$ Jersey $+1 / 8$ PS $+1 / 8$ Brahman & 22 & $3 / 8$ Reyna $+5 / 16$ Jersey $+5 / 16$ PS \\
11 & $1 / 4$ Reyna $+3 / 4$ PS & 23 & $5 / 8$ Reyna $+3 / 8$ Brahman \\
12 & $1 / 4$ Reyna $+3 / 8$ PS + 3/8 Brahman & & \\
\hline
\end{tabular}


Cualidades y defectos del Reyna. Al Reyna se le atribuyen diferentes bondades cuando se explota tanto en hatos puros como en cruces con razas especializadas, señalando los ganaderos las siguientes.

- Mantiene buena condición corporal durante el verano

- Buena rusticidad

- Buena persistencia en producción

- Mejora la fertilidad, resistencia y reducción de los intervalos entre partos en cruces con razas especializadas

- Temperamento manso

- Producción de leche aceptable

- Terneros con manejo adecuado se destetan entre los nueve y 10 meses con $180 \mathrm{~kg}$ de peso vivo

- Leche de buena calidad

- Toros presentan buena libido

- Resistencia a garrapatas

- Resistente a enfermedades de las pezuñas

- Resistente a manejos deficientes

- Animales de buen desarrollo

- Resistentes al calor

- Sobrevive con pastos naturales

- Buen rendimiento en carne en el matadero

- Son animales muy precoces

- Suavidad al ordeño y chorro recto

A pesar de las ventajas que se puedan señalar del ganado criollo, también los productores señalan algunos aspectos adversos que si los cuantificamos, no superan los aspectos positivos ya señalados.

Dentro de estos aspectos se señalan los siguientes.

- Defecto de la pelvis en la región caudal en algunos animales (Grupa caída)

- Reyna puro es de cuernos débiles que no es favorable para el propósito de bueyes

- Lento desarrollo en peso durante el primer año de vida

- Tamaño mediano de los adultos

- Hembras no son altas productoras de leche

- Probabilidad de mucho parentesco entre toros

- En algunas zonas pueden tener dificultades para su mercadeo

\section{CONCLUSIONES}

Nadie puede negar o poner en duda la influencia que ejercieron las razas criollas en la economía del país y en el desarrollo de la ganadería hasta los años 1950.

El Reyna como sobreviviente único de las razas criollas, tiene presencia en once de los dieciséis departamentos del país tanto en hatos puros como en los diversos cruces en que participa, alcanzando una distribución irregular en los diferentes departamentos en los que se contabilizó su presencia, con sus mayores poblaciones en los departamentos de Rivas y Boaco. Tal distribución irregular puede estar influenciada por la poca difusión y estrategias de venta por parte de los criadores del hato puro, que no han sabido vender las virtudes de esta Raza a los demás ganaderos del país.

La mayoría del hato Reyna se explota en sistemas de producción de doble propósito con alimentación basada principalmente en pastizales y con una excepción en un sistema semiintensivo atípico, en el que se le suministra suplementación.

La calidad de la leche del Reyna es muy buena en relación a otras razas, revelando valores de proteína que fluctuaron en este estudio entre $2.8 \%$ y $4.16 \%$ y un contenido de grasa que varía entre $1.8 \%$ y $4.6 \%$.

El ganado criollo se ha cruzado con diferentes razas encontrándose en este caso en un total de 23 grupos raciales, acompañando a las razas Brahman, Pardo Suizo, Jersey, Simmental, Brahman y Gyr, dominado las proporciones de media sangre y un cuarto Reyna.

Dentro de todas las ventajas señaladas sobre el Reyna, las más importantes en cuanto al impacto que tienen sobre la mayoría de los índices productivos están, la reducción que sufren los intervalos entre partos cuando estas se cruzan con razas europeas de alta especialización, la mejora en la precocidad y fertilidad de las hembras, y en general el logro que se adquiere en el fortalecimiento de la productividad de los hatos.

El ganado Reyna como bovinos naturalizados en Nicaragua, representan un potencial genético de incalculable valor, sometido a más de 500 años de selección natural y por lo que debe ser de interés de todos, el conservarlos y multiplicarlos para evitar su reducción poblacional o desaparición. 


\section{REFERENCIAS BIBLIOGRÁFICAS}

Esgueva Gómez, A. 2006. Nicaragua en los Documentos, Tomo I, 1523 - 1857, Instituto de Historia de Nicaragua y Centroamérica. Primera edición. Managua, NI.

FAO (Organización de las Naciones Unidas para la alimentación y la agricultura, IT). 2005. El estado de los recursos zoogenéticos de Nicaragua. p 19.

Guerrero, J, Soriano, L. 1992. Historia de la ganadería de Nicaragua. ed. Unión de Cardoza y CIA. Ltda. Managua. NI. 187 p.

Incer Barquero, J. 1969. Geografía de Nicaragua. Librería y editorial Recalde, S.A. Managua. NI. p 212 - 213.

Levy, P. 1976. Notas geográficas y económicas sobre la república de Nicaragua. ed. Fondo de promoción cultural del Banco de América. Managua, NI. 563 p.

Primo, AT. 1992. El ganado bovino ibérico en las américas, 500 años después. EMBRAPA/CPATB. RS. BR. Arch. Zootec. 41 (extra): 421-432

Rouse, JF. 1977. The Criollo, Spanish Cattle in the Americas. Universíty of Oklahoma Press, Norman, p. 303. 